\title{
La escena urbana como estrategia apostólica. Un aporte trunco de la Compañía de Jesús para Montevideo
}

Urban scene as apostolic strategy. A truncated contribution from the Company of Jesus to Montevideo

\author{
Norberto Levinton ${ }^{*}$
}

Resumen: La idea de este trabajo es que la Compañía de Jesús implementó el proyecto de una iglesia y colegio para Montevideo en un lugar preferencial con el objetivo de poner en valor la identidad cultural de los vecinos e incorporarlos a una vida cristiana "para mayor gloria de Dios". La elección del sitio dando marco a la plaza principal y enfrente del cabildo constituyó, desde nuestro punto de vista, una estrategia apostólica. Para sustentar esta conceptualización analizaremos el proyecto arquitectónico propuesto, el perfil cultural de los vecinos y la estructura de la ciudad.

Para ello incluiremos este diseño arquitectónico comparativamente dentro de una serie de proyectos y obras del mismo coadjutor arquitecto de la Compañía de Jesús, incursionaremos en la arquitectura mudéjar canaria y daremos cuenta de la traza urbana tomando como referencia una pintura realizada algunos años después.

Palabras claves: mudéjar, significante, significado, mensaje y ciudad.

\begin{abstract}
The idea of this work is that the Society of Jesus implemented the project of a church and school to Montevideo in a special place with the aim of putting the cultural identity of residents in value. Site selection framework giving the main square and opposite the town hall constituted, from our point of view, was an apostolic strategy. To support this conceptualization we ill analyze the proposed architectural project, the cultural profile of the neighbors and the structure of the city.
\end{abstract}

\footnotetext{
*Arquitecto y Doctor en Historia Universidad del Salvador. E-mail: n.levinton@gmail.com. Argentina.
}

91 Norberto Levinton. La escena urbana como estrategia apostólica... 91 a 106. 
This architectural design will be include comparatively in a series of projects and works by the same architect coadjutor of the Society of Jesus, We will introduce on the Canary . Mudejar architecture and will analyze the urban plan of Montevideo to use like reference a painting done some years later and others similar experiences.

Keywords: Mudejar, meaningful, meaning, message and city.

Recibido: 19 de julio de 2015

Evaluado: 10 de octubre de 2015 
El resultado del entrecruzamiento de las fechas: la fundación (dar inicio) de Montevideo, la llegada de los habitantes, el arribo de los jesuitas a la ciudad y la producción del proyecto arquitectónico por el hermano coadjutor Antonio Forcada

En noviembre de 1723 los portugueses se retiran de la zona de la Bahía de Montevideo. Inmediatamente los españoles construyen en 1724 un baluarte militar. Domingo Petrarca, Capitán de Ingenieros, define las primeras 6 manzanas de la futura ciudad.

El Capitán de Corazas español Pedro Millán traza el plano con el damero y los límites. El 24 de diciembre de 1726 se realiza la repartición de solares de la ciudad. Millán indica los lugares más adecuados para los edificios de carácter público ${ }^{1}$. El plano de 1730 ya presenta la Plaza Mayor con una ubicación jerárquica (Fig. 1). En 1739 los jesuitas piden al Gobernador Salcedo que se les de "dos cuadras" (dos manzanas) de terreno. El Cabildo de Montevideo se las adjudica. Sin embargo al poco tiempo deben cederlas porque la construcción de la muralla requiere ese espacio. Es el año 1740. Montevideo es una población-fortaleza. Una Real Cédula del 9 de diciembre de 1743 ordena que se ayude a los jesuitas. En 1744 los vecinos se presentan al Rey solicitando fondos del tesoro real para el establecimiento de los jesuitas, esto dado la pobreza de la ciudad. Hay interés de los ciudadanos porque quieren un buen colegio para sus hijos. Otra Real Cédula del 31 de diciembre de 1744 autoriza el establecimiento definitivo de la Compañía de Jesús. Forcada pasa por Montevideo, el barco de la misión del Padre Rico se detiene por poco tiempo, y llega a Buenos Aires el 15 de julio de 1745. Santos de Uriarte, el Comandante de Montevideo, ofrece donar diez mil pesos, el 27 de septiembre de 1745, para construir la Residencia de los jesuitas. En Córdoba, lugar de residencia del Padre Provincial, la Compañía

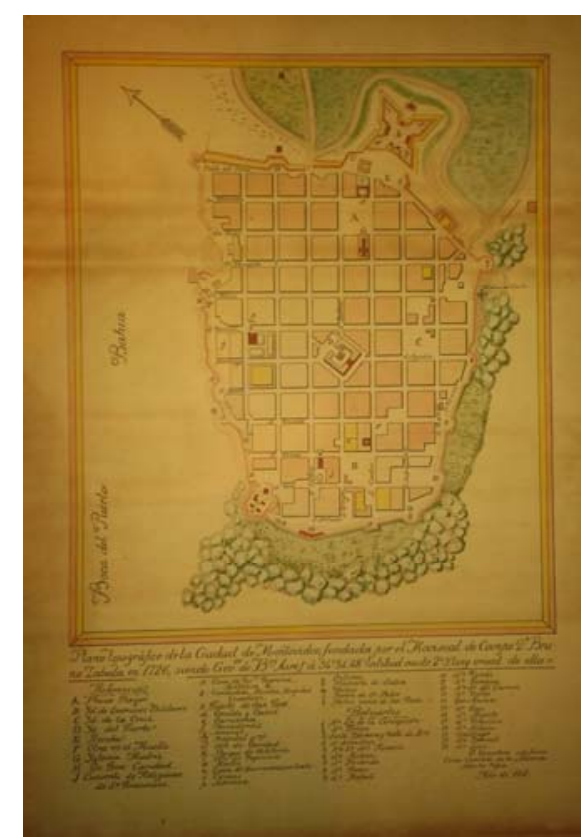

Fig. 1 Ciudad de Montevideo a mediados del siglo XVIII. Archivo de Montevideo. de Jesús acepta la donación por medio de una escritura realizada el $1^{\circ}$ de diciembre de $1745^{2}$. El 3 de octubre de 1747 se efectiviza la donación recibiendo los jesuitas por la mitad: diez mil pesos, diez mil reses a 3 pesos cada una y veinte mil pesos de plata. Por la otra mitad solares, casas y algunos esclavos negros. Los jesuitas se instalan temporalmente en una casa que había sido de Uriarte donde crean la Residencia de San Estanislao de Kostka $^{3}$.

En 1749 compran los jesuitas un sitio en una esquina principal lindando al sur con la Plaza Mayor con 50 varas de frente a ella. Necesitaban toda la manzana y la van

\footnotetext{
${ }^{1}$ Carmona/Gómez, 1999.

${ }^{2}$ Ferres, 1975: 33.

${ }^{3}$ Ferres, 1975: 41. Hoy es la zona de la plaza Zavala.
} 
adquiriendo de a poco. Forcada, el 5 de octubre de 1749, sabemos que estaba en Córdoba por la certificación de los últimos votos ${ }^{4}$. Estaba dedicado a varias obras. Entre ellas partes de las iglesias de la estancia de Santa Catalina (cúpula y cuerpo de la iglesia), la estancia de Alta Gracia (fachada con trazas mixtilíneas y atrio semi octogonal), habitaciones de servicio para el Colegio Máximo (aparecen en el plano hecho por él), Jesús María (cúpula gallonada). Por ello suponemos que hizo en Córdoba el proyecto para Montevideo de manera análoga a como hizo Bianchi el proyecto para la iglesia de San Miguel (actual Brasil) y que ejecutó in situ el Maestro de Obras Prímoli. Forcada, también intervino de la misma manera en el proyecto del edificio de la estancia del Río de las Vacas o de La Calera, edificio finalmente construido por el Hermano Schmidt.

\section{El mudéjar como lenguaje arquitectónico del hermano Antonio Forcada}

El P. Forcada ingresó a la Compañía de Jesús en 1735. Su primer trabajo fue para intervenir en la iglesia del Colegio Fontinense de Alicante. Este colegio es analizado por Gómez-Ferrer ${ }^{5}$ y esta historiadora menciona que en la época que estuvo Forcada se construyó una de las alas del claustro con columnas toscanas. La cúpula es ochavada y gallonada. Son elementos arquitectónicos de un tipológico lenguaje regional de los jesuitas en Aragón. La resolución del Colegio de Alicante aparece en el plano traído al Río de la Plata por Forcada lo que permite asegurar que es el autor del proyecto.

En Ontinyent (Valencia) las obras del Colegio y de la Iglesia se inician en 1736. Forcada estuvo allí desde 1737 a 1740 por lo que suponemos que tuvo una importante incidencia en el proyecto $\mathrm{y}$ en los trabajos de movimiento de tierras, cimentación y ejecución de los muros de mampostería ${ }^{6}$. Así también opina Gómez-Ferrer ${ }^{7}$. De allí fue al Colegio de San Sebastián y San Francisco de Borja de Gandía. Gómez-Ferrer le atribuye a Forcada el diseño de la planta. La cúpula es gallonada y tiene un tambor octogonal. El presbiterio es ochavado. Hubo un terremoto en 1748 por lo que el plano de Forcada se constituye como un documento fidedigno de cómo iba a ser la iglesia e incluso contiene partes que finalmente no se hicieron.

En lo que respecta a Alagón también se cree que Forcada proyectó la iglesia de San Antonio $^{8}$. A la entrada hay un arco mixtilíneo y la cúpula es gallonada. Siempre aparecen en todas estas obras el diseño de figuras ochavadas.

Carretero analiza la intervención de Forcada en el colegio jesuítico de Tarazona durante 1741. Su paso por este edificio a primera vista no parece haber sido muy importante. La autora le atribuye la ejecución de habitaciones de servicio que se hicieron cuando estuvo Forcada: nuevo refectorio, cocina, librería y ropería. Empero vemos que en

\footnotetext{
${ }^{4}$ Storni, 1980: 104

${ }^{5}$ Gómez-Ferrer, 2012: 391.

${ }^{6}$ Navarro Catalán, 2012: 372. El desarrollo de la mampostería en esa época está confirmada por este autor. Carretero, 2012: 161.Le atribuye la iglesia a Forcada.

${ }^{7}$ Gómez-Ferrer, 2012: 382. Ver figura 20, uno de los planos de Forcada.

${ }^{8}$ Vázquez Barrado, 1994. Ibáñez Fernández/ Criado Mainar, 2012: 421. Ver la figura 22.
}

94 Norberto Levinton. La escena urbana como estrategia apostólica... 91 a 106. 
la serie de planos publicados por Furlong hay invariantes regionales que pudieran haber sido proyectados por Forcada. Nos ha llamado la atención en la iglesia de San Vicente Mártir la implementación de pilastras toscanas ${ }^{9}$. Carretero señala que el toscano abarata sobremanera la fábrica debido a su escasez de talla ${ }^{10}$. Esto permitiría hacernos pensar que Forcada fue el autor de las pilastras de ese estilo en Santa Catalina. O sea que la elección de la tipología constructiva, que alguno atribuye a Bianchi ${ }^{11}$ ya que este también utiliza el orden toscano, pudiera haber tenido que ver con el costo de los trabajos y quizás con la escasa ductilidad de la mano de obra. Hay otras soluciones utilizadas permanentemente en la arquitectura conventual de Tarazona como las bóvedas de cañón con lunetos que también implementa Forcada. La cúpula del templo jesuítico de San Vicente Mártir, obra de mediados del siglo XVIII, es gallonada, se dispone sobre pechinas y permanece abierta por ocho vanos cuadrangulares separados por una decoración en yeso formada por parejas de pilastras salomónicas y ensartos de flores que se aglutinan en la clave. Carretero afirma que la utilización de este tipo de cúpula fue una solución muy ingeniosa que ya recogía el religioso valenciano Tomás Vicente Tosca en su "Tratado de Arquitectura civil, montea, cantería y relojes", publicado en 1712. Por su parte Azanza López asegura que la cúpula gallonada o alunetada se empleó sobre todo en la arquitectura castellana, andaluza, aragonesa y valenciana de mediados del siglo XVIII ${ }^{12}$. Forcada utiliza esta cúpula en Jesús María y en Santa Catalina. Carretero atribuye al hermano José Galbán (estuvo en Tarazona entre 1748 y 1750) muchas decisiones arquitectónicas que pudieran haber sido de Forcada como el hastial de perfil mixtilíneo. Hay una gran similitud entre uno y otro con respecto a su extracción regional y su formación ${ }^{13}$. De Forcada se hace mención en el plano de Tarazona. Allí se señala con el $\mathrm{N}^{\circ} 14$ toda un ala de edificio y se dice que es "obra que se ideó por el Hermano Antonio Forcada que no se ha hecho en marzo del 41".

El último trabajo de Forcada en España fue en Calatayud donde proyectó el Seminario de Nobles (inaugurado en 1752) y también rediseñó la iglesia de Nuestra Señora del Pilar, actualmente conocida como San Juan el Real. En 1748, Forcada ya no estaba, se ejecutó el transepto, la capilla Mayor y la sacristía. La cúpula es gallonada. Para Ibáñez Fernández/Criado Mainar "debieron usarse unas nuevas trazas confeccionadas por el

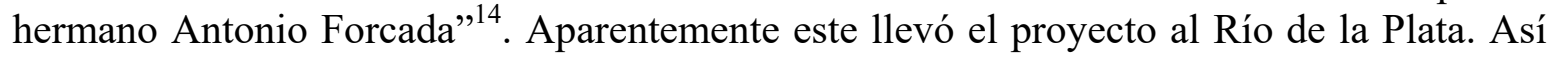
lo demuestra la colección de planos encontrada por Furlong.

En septiembre de 1742 Forcada ya estaba comprometido con la Provincia del Paraguay. En el libro de gastos del procurador, el Padre Rico, aparecen los costos de las

\footnotetext{
${ }^{9}$ Carretero, 2012: 56.

${ }^{10}$ Carretero, 2012: 177.

${ }^{11}$ Furlong, 1946: 218. "El barroco de la airosa y bella fachada del templo contrasta con el clasicismo impecable del claustro, obra esta última de Blanqui o de Prímoli, en cuanto a los planos, aunque construido después por algún otro jesuita".

${ }^{12}$ Carretero, 2012: 57.

${ }^{13}$ Carretero, 2012: 64. Galbán nació en Uncastillo (Zaragoza) en 1705 e ingresó en 1737 a la Compañía de Jesús. Fue Coadjutor Temporal en 1746.Trabajó en Huesca entre 1742 y 1746, en Calatayud de 1746 a 1748 y luego en Alagón. Forcada nació en Nuez del Ebro (Zaragoza) en 1701. Ingresó en 1735 en la Compañía de Jesús.

${ }^{14}$ Ibáñez Fernández/ Criado Mainar, 2012: 407.
} 
herramientas del Hermano Forcada. Para noviembre Forcada se trasladó de Zaragoza a Madrid. En diciembre se le preparó un vestuario. Al año siguiente, en mayo, Forcada estuvo en el Paular (cerca de Segovia) para interiorizarse sobre la fabricación de papel. Para septiembre de 1744 viajó de Madrid al Puerto de Santa María. En noviembre se hicieron instrumentos de herrería y carpintería. La descripción de las herramientas es muy interesante porque es posible vincular este material con lo hecho en las obras de Jesús y San Cosme (Paraguay) $)^{15}$.

Su barco hacia América zarpó el 23 de marzo de 1745. Pasó por Montevideo y arribó a Buenos Aires el 15 de julio. El primer trabajo que realizó fue en Santa Fe: rediseñó la fachada de la iglesia de la Compañía de Jesús. Así lo indican los arcos con escotaduras muy similares a los de la fachada de la iglesia de la estancia de Alta Gracia. Allí Furlong encontró una importante serie de planos, algunos españoles. En varios hubo participación comprobada de Forcada. Luego se trasladó a Córdoba. De aquí hay escasa documentación. Un memorial del Padre Nussdorfer indica la existencia de trabajos en Alta Gracia poco tiempo antes de su arribo ${ }^{16}$. También se estaban realizando obras en Jesús María. La presencia de Forcada en las rejas con motivos mixtilíneos ya lo hemos mencionado en trabajos anteriores ${ }^{17}$. Los mismos motivos aparecen en Jesús María, Alta Gracia y en el Convento de Santa Teresa de Jesús ${ }^{18}$. Furlong lo menciona en la década del 40'y Nadal Mora en la década del $50^{\prime 19}$. En otros edificios se puede adjudicar la imitación de las rejas a Jaime Roca. El lenguaje arquitectónico de Forcada aparece nítidamente en la fachada de la Estancia de Alta Gracia. Tiene tres cuerpos con entablamento mixtilíneo. Los tres cuerpos verticales presentan una partición evidente: un cuerpo central levemente retrocedido, sobreelevado en dos niveles y contenido por pares de pilastras toscanas pareadas monumentales que comparten un basamento complejo y sostienen un frontis mixtilíneo quebrado y dos cuerpos laterales de menor altura ${ }^{20}$. En la calle central la puerta de ingreso también posee un arco escarzano con escotaduras levemente abocinado por moldura tipo listel. La ventana remata en otro frontón mixtilíneo. El atrio curvo, elevado al modo barroco, la separa de su entorno inmediato y es ochavado.

Sobre Jesús María. el Padre Sobrón ya había indicado que los elementos decorativos del exterior de la cúpula señalarían una vinculación con el autor de la Iglesia de Santa

\footnotetext{
${ }^{15}$ AGNA. Sala IX, 7-1-1. S/año. División Colonia. Sección Gobierno. Compañía de Jesús. Varios años. "Encargo que hace a Don Joseph de Villanueva Pico el Padre Juan Joseph Rico y le suplica completar para su Provincia del Paraguay lo siguiente: primeramente 30 quintales de hierro, planchuela y mitad y mitad según las muestras que van ahí incluidas en carta del Padre Forcada pues son para unos instrumentos suyos y que él sabe manejar en Indias". La explicación está en los cajones enviados: "Memorial de lo que va en los 4 cajones que llevan en sus calesas el Padre Boxer con sus compañeros. En otro cajón casi cuadrado van 20 escuadrías de hierro para canteros".

${ }^{16}$ AGNA Sala IX 6-9-7 Memorial del Padre Nussdorfer para la estancia de Alta Gracia 25 de diciembre de 1745. "y se dará algún buen revoque así a la bóveda de la sacristía".

${ }^{17}$ Levinton, 1999:193

${ }^{18}$ AGNA, Sala IX, 6-9-7. Memorial del Padre Machoni para Alta Gracia. "se acabará la herrería empezada".

${ }^{19}$ Furlong, 1946; Nadal Mora, 1957.Ver también Page, 2000:111.

${ }^{20}$ AECI, 2004:9 y 10.
} 
Catalina. Desde mi punto de vista la cúpula gallonada es indiscutiblemente una expresión típica del mudéjar aragonés.

En 1750 se piensa que se inició la obra de la iglesia de la estancia de Santa Catalina. Para mí, salvo detalles de la fachada, es indudablemente obra de Forcada. Pellet Lastra se refiere al estilo de la obra como "Churrigueresco" o relativo al barroco español. Señala los detalles constructivos de la Sacristía con sus pilares ochavados, la cúpula y "ciertos detalles de herrería" ${ }^{21}$. En una última visita me impresionó el remate en forma piramidal del interior del chapitel como una expresión del mudéjar aragonés. Se parece al sistema de bovedillas por aproximación de hiladas

El Padre Grenón atribuye la hechura, de Forcada según Carretero, del plano de Colegio Máximo al año 1748. Gallardo menciona la existencia de un arco mixtilíneo en la fachada de Las Teresas con el año 1753 inscripto por la conclusión de la obra ${ }^{22}$. Furlong asevera que Forcada estaba en Córdoba en $1753^{23}$. Para Page durante 1754 se trabajó en el Colegio Máximo donde "se ha hecho ángulo nuevo de la portería principal, ha hechóse también cocina, despensa y contra-despensa todo de bóvedas, con su tránsito",24.

Buschiazzo afirma que datan de junio de 1754 las primeras anotaciones de construcciones en un libro de gastos correspondiente a la estancia de Santa Catalina. Las anteriores anotaciones habían sido en 1718. Sin embargo describe la sacristía de la iglesia.: "con una pequeña cúpula que recae sobre pechinas que a su vez descansan sobre pilares ochavados o emboquillados... Esta bellísima sacristía podría emparentarse con la de Jesús María, lo mismo que la cúpula del templo y ciertos detalles de herrería"25.

Pero en otro texto Buschiazzo aclara: "Pero aun cuando la primera anotación importante data de la fecha indicada más arriba, es evidente que las obras debieron comenzarse varios años antes, pues hacia esa misma época se registran gastos de "42 espejos de 4 ps y 224 vidrios a $21 / 2$ rs", varias entradas de oro destinadas al dorado de los altares y 2 arañas de plata, indicios todos de que el templo estaba en condiciones de habilitarse al culto" ${ }^{26}$.

Desde mi punto de vista el texto leído por Buschiazzo permitía otra interpretación. Dice en un párrafo: "En este tiempo se han hecho varias obras en nuestra estancia y se está trabajando en la capilla, la que se espera será muy hermosa con su crucero"27.

Lo que definiría una datación más amplia. Forcada de Córdoba pasó a Corrientes donde, hacia 1755, proyectó la iglesia de la Compañía de Jesús. Suponemos que estuvo en Asunción del Paraguay donde intervino en el Colegio y en la Iglesia. En 1759 estaba en

\footnotetext{
${ }^{21}$ Pellet Lastra, 1917.

${ }^{22}$ Gallardo, 1978.

${ }^{23}$ Furlong, 1946: 222.

${ }^{24}$ Page, 1999: 73.

${ }^{25}$ Buschiazzo, 1969.

${ }^{26}$ Buschiazzo, 1940:10.

${ }^{27}$ Lascano González, 1941: 71.
} 
Jesús y San Cosme y San Damián. Ambos pueblos tenían decidido su traslado a un nuevo asentamiento.

En San Cosme están los pilares ochavados del Colegio. El trabajo de la madera es típicamente mudéjar. Dice Busaniche sobre la herrería: "una hermosa reja de hierro batido, cuyos hermosos dibujos nos recuerdan las rejas jesuíticas de Santa Catalina, en Córdoba" ${ }^{28}$.

En Jesús el Colegio también tiene pilares ochavados. En cambio la iglesia presenta gruesos pilares flanqueados de otros pilares, según define Golvin, "enganchados"29.

Lugones menciona los sillares a medio desbastar y las junturas desbordando aun la $\operatorname{argamasa}^{30}$. Busaniche, en cambio, señala que los "sillares están muy bien recuadrados"31. Para eso Forcada traía los moldes desde España.

El arco mixtilíneo de la fachada de la iglesia es significativo. El perfil tiene un intradós formado por líneas mixtas guardando una perfecta simetría. En el baptisterio se encuentra una pequeña cúpula apoyada sobre trompas, detalle curioso y poco frecuente en América pero muy usado en Teruel (España), zona del mudéjar almohade, para campanarios. El arco de "cortinado" que compone la entrada a la sacristía, trazado por dos porciones de circunferencia con centros exteriores que se cortan en la clave formando ángulo, puede ser entendido como una posible asimilación de estructuras góticas al sistema de trabajo mudéjar, así lo define Borras Gualis, o siguiendo el pensamiento de Lambert se trataría de una forma cuya genealogía era hispano-musulmana. En el caso de los estípites, o sea las pilastras que tienen la figura de una pirámide truncada con la base menor hacia abajo, hay muchos ejemplos de su uso en Aragón en la arquitectura jesuítica de Zaragoza. También en San Francisco Xavier de Tepotzotlán en México.

Forcada en 1762 fue consultado para intervenir en el templo de San Ignacio Miní. Tuvo un accidente en San Cosme, se cayó de un andamio, del cual no se recuperó totalmente.

Murió el 30 de junio de 1767 en San Ignacio Miní.

\section{La procedencia mayormente canaria de los habitantes de Montevideo ${ }^{32}$. El mudéjar en las Islas Canarias.}

El mudéjar andaluz fue dominante en Canarias debido a que esa fue la procedencia de los colonos que fueron a estas islas en los siglos XV y XVI. La población guanche era berebere. Por lo cual había influencias almorávides y almohades como tenía la arquitectura regional aragonesa realizada por Forcada. La iglesia de la Concepción de Santa Cruz de Tenerife fue reconstruida en 1653. Su cubierta interior es un artesonado mudéjar. La iglesia

\footnotetext{
${ }^{28}$ Busaniche, 1955: 85.

${ }^{29}$ Golvin, 1970.

${ }^{30}$ Lugones, 1973.

${ }^{31}$ Busaniche, 1955: 107.

${ }^{32}$ También hubo algunos gallegos.
} 
de la Concepción de San Cristóbal de la Laguna también tiene un artesonado mudéjar. La basílica de Nuestra Señora del Pino tiene una torre de planta octogonal.

Canarias tuvo períodos de gran producción de azúcar y luego de gran explotación vitivinícola. Hacia 1688 la población superaba los 105 mil habitantes. Pero la exportación de vino a las colonias inglesas fue perjudicada por la producción portuguesa. Portugal e Inglaterra ya habían constituido su poderosa alianza. Entre 1701 y 1713 la guerra de la sucesión española tuvo consecuencias en el Río de la Plata. España tuvo que devolver la Colonia del Sacramento. España tenía que reforzar sus fronteras. Por eso el Reglamento Real de 1718 fijaba la obligación de enviar cinco familias a América por cada 100 toneladas de mercancías exportadas. De esta manera se conciliaba la necesidad de la Corona española con las urgencias de los canarios.

Manuel de Freytas Fonseca fundaba, para Portugal, Montevideo el 22 de noviembre de 1723. Se quedó solo dos meses siendo desplazado por los españoles. El gobernador español Zavala tenía la orden de fortificar la península donde iba a estar la ciudad de Montevideo.

En 1726 hubo un primer viaje de inmigrantes canarios a Montevideo. Un segundo viaje de inmigrantes canarios se efectuó en 1729. Estos colonos participaron de los repartos de tierras en la ciudad de Montevideo.

\section{El proyecto del Hermano Forcada}

La planta de San Lorenzo el Real de Burgos (España) es en cruz griega y conforma un volumen ininterrumpido de suerte que se forma un circuito para la serie de capillas dispuestas en sus muros en torno al ámbito central (Fig. 2). Unas bóvedas enriquecidas con elaboradas yeserías cubren el deambulatorio mientras en el centro, sobre gruesos pilares de sección octogonal, se alza una gran cúpula gallonada e iluminada por una linterna. Para Chueca Goitía: "La Iglesia de San Lorenzo en Burgos....dentro de la organización central frecuente en las iglesias de la Compañía de Jesús, conserva un sentido estructural gótico, comprensible en una ciudad donde este estilo tuvo tanta preponderancia. De un ochavo con capillas cuadradas, se pasa a una cúpula sobre cuatro pilares. Construcción ligera y atrevida donde no faltan los arcos apuntados y el recuerdo de los ochavos del gótico tardío. Capillas ochavadas, traducidas al barroco....soluciones y formas del ayer con nuevos ropajes" ${ }^{33}$.

En el trabajo hecho por Furlong y Buschiazzo el plano $\mathrm{n}^{\circ} 3$ dice "Colegio de Montevideo que no se llegó a construir ${ }^{34}$ (Fig. 3). Asimismo el plano $n^{\circ} 4$

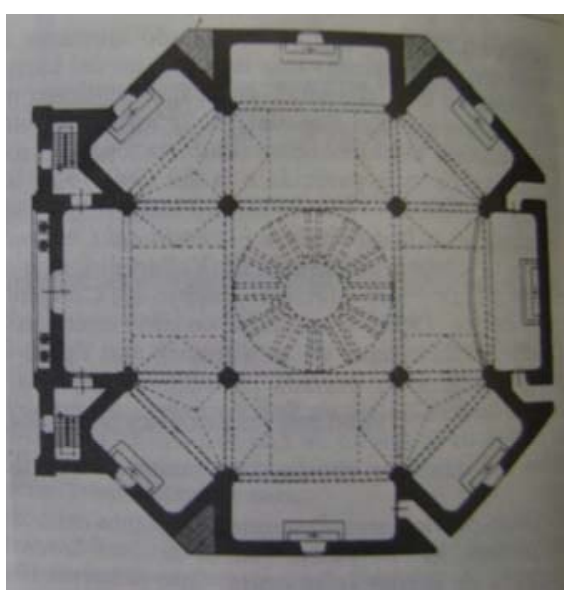

Fig. 2 Iglesia de San Lorenzo en Burgos (España)

\footnotetext{
${ }^{33}$ Chueca Goitía, 1985: 61.

${ }^{34}$ Furlong/Buschiazzo, 1943: 465.
} 
(Fig. 4). La letra en ambos es de Forcada. El primero tiene una cúpula de gallones como las obras de Córdoba que ya mencionamos y atribuimos a Forcada. Los autores resaltan la composición central y la forma octogonal que "recuerda a la iglesia de los jesuitas de San Lorenzo en Burgos". En cuanto al segundo tiene una escala en varas y mide 100 varas lo que implica, como dicen Furlong/Buschiazzo, que está tomando dos manzanas separadas por una calle. Esto coincide con lo que acota Ferrés sobre el uso de la otra manzana para la

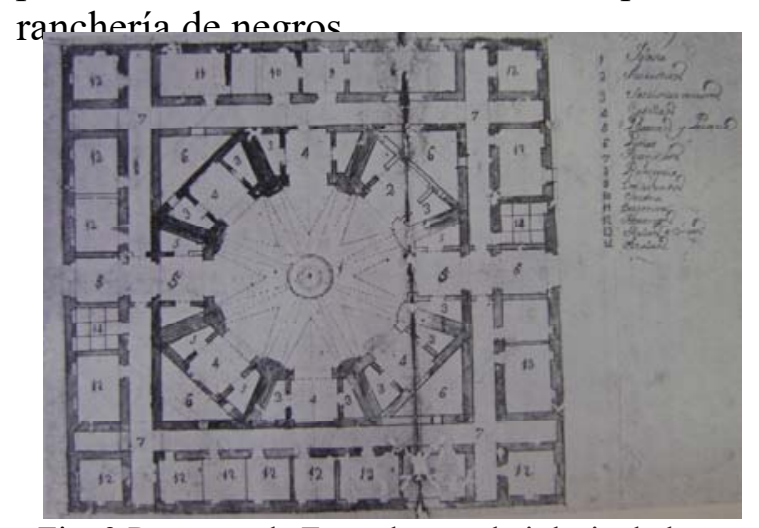

Fig. 3 Proyecto de Forcada para la iglesia de la Compañía en Montevideo similar al de Burgos

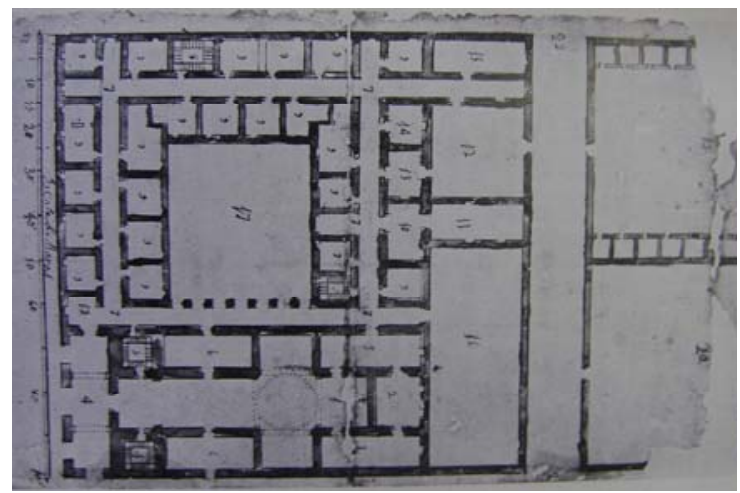

Fig. 4 Proyecto alternativo hecho por Forcada.

Dice Furlong sobre el proyecto: "recuerda a la iglesia de los Jesuitas, de San Lorenzo, en Burgos, si bien en esta la cúpula apoya sobre cuatro columnas, en tanto que en el proyecto montevideano arranca directamente de los muros perimetrales. El otro proyecto nos muestra un conjunto de edificación que cubriría dos manzanas, separadas por una calle. En una de ellas estaría el templo, en forma de cruz latina, con dos torres y dos grandes capillas laterales completamente separadas de la nave única del templo, exactamente igual que la Compañía de Córdoba"35.

En 1763 el Colegio de Montevideo estaba lleno de deudas, lo que habría impedido la construcción de lo proyectado por Forcada ${ }^{36}$.

\section{El lugar elegido por los jesuitas. La traza de la ciudad de Montevideo en la pintura de Brambilla.}

Para Baracchini/Altezor la calidad de ciudad amurallada mediterránea de Montevideo, donde se privilegió lo militar, niega el carácter de la bahía como puerto natural, no cumple con las disposiciones de las Leyes de Indias y la convierte en una ciudad que prioriza el hinterland. La Plaza Mayor fue ubicada en el lugar más alto, central bastante próximo al portal de ingreso al recinto. El esquema ortogonal del damero de calles

\footnotetext{
${ }^{35}$ Furlong, 1946: 140.

${ }^{36}$ Ibíd.: 227.
} 
rectilíneas no se altera en la Plaza Mayor con manzanas que medían 83,6 m (100 varas) y las calles $10 \mathrm{~m}$ de ancho (Fig. 5).

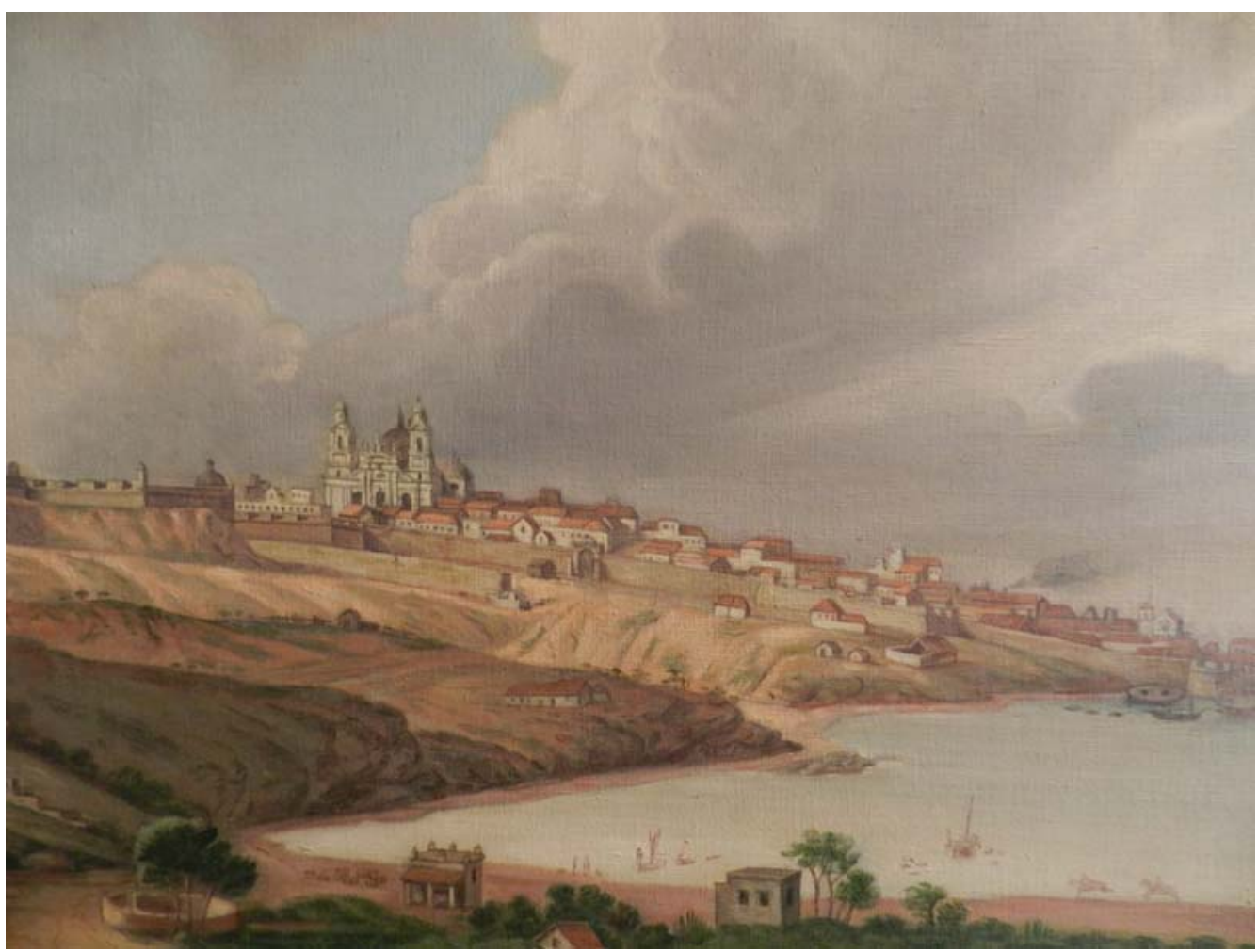

Fig. 5 Pintura de Montevideo. Museo Casa de Rivera.

En 1746 regresaron los jesuitas a Montevideo y en 1749 levantaron en la esquina nordeste, de la intersección de las actuales calles Ituzaingó y Rincón, una pequeña capilla de 15 por $4,20 \mathrm{~m}$ y $4,70 \mathrm{~m}$ de altura, con techo de tejas, a dos aguas y con una pequeña espadaña con su campana. Los padres ocupaban, escribe Giuria, el resto de la fachada sobre la calle Rincón y delante de ellos estaba dispuesta una galería de regular anchura, que daba a un patio de no gran amplitud. Además había otros locales destinados a cocinas, refectorios, habitaciones de servicio y otros. La escuela funcionaba en las salas que daban frente a la calle Ituzaingó y dichas salas estaban iluminadas desde la vía pública y de un vasto jardín por medio de varias ventanas de regulares dimensiones ${ }^{37}$.

Para Ferrés: "los proyectos de los Padres iban muy lejos. Tenían la intención de construir un Colegio que pudiera rivalizar con los mejores de la Provincia; junto a él edificarían una iglesia de subido valor arquitectónico... para lo cual habían ya preparado algunos planos" ${ }^{38}$.

El proyecto realizado por el Hermano Coadjutor Arquitecto Antonio Forcada tenía una impronta pensada para Montevideo. Según Ibáñez Fernández/Criado Mainar todos los

\footnotetext{
${ }^{37}$ Furlong, 1946: 139.

${ }^{38}$ Ferres, 1975: 46.
} 
edificios jesuíticos de Aragón -donde intervino Forcada- "tuvieron una clara vocación urbanística, ya que trataron de ordenar el espacio urbano en el que se inscribieron" ${ }^{\natural 9}$.

En el momento de la expulsión de la Compañía de Jesús en 1768 tenían en la manzana frente a la plaza principal: "Un sitio de 50 varas de frente y 50 de fondo y fabricado en el una casa de piedra...hace frente a dos calles...la Casa en la que viven los sujetos su sitio es de media cuadra entera hace frente a 2 calles...tiene portería y puerta seglar...en el patio 4 aposentos en que viven los sujetos...Iglesia, sacristía, refectorio, escuela, cocina y otras oficinas...tiene ranchería para los esclavos una cocina de adobe...la iglesia tiene un altar mayor y otro altar colateral ${ }^{30}$.

Benedetti habla de la arquitectura de la Compañía de Jesús "come strumento imperituro di gloria per l'uomo per la familia per la città"41. Sale, por su parte, agrega la idea de la "scelta del sitio come strategia apostolica" porque la orden pensaba abocarse al apostolado citadino... stabilirsi al centro della città, in un luogo cioè cosi strategicamente significativo per il potere religioso e civile [el lugar para il Gesú de Roma]”,

Estos conceptos se ven reflejados en las acciones concretas. En el caso de Salamanca la conformación urbana del núcleo primitivo de la ciudad era tal que se elevaba sobre la loma central que queda entre las vaguadas de los arroyos de los Milagros y de San Pablo ${ }^{43}$. La Clerecía, Colegio Real de la Compañía de Jesús, fue asentada sobre la colina de San Isidro. Desde allí dominaría junto con las Catedrales el panorama entero de la ciudad ya que tendría ante sí todo el valle. Fue fundación de los reyes de España y la reina, por sugerencia de su confesor un padre jesuita, exigió que estuviera en lo más céntrico ${ }^{44}$. El 22 de diciembre de 1616 Felipe III escribió al Municipio: "el edificio ha de ser para tanto ornato y lustre de esa ciudad y de la doctrina y letras que en el se ha de profesar, de que se ha de seguir tan gran servicio de Nuestro Señor y bien de esa república" ${ }^{45}$.

Otro ejemplo es Buenos Aires, donde los jesuitas tuvieron su primer emplazamiento junto al fuerte. Es decir que el colegio de los jesuitas estaba frente a la Plaza Mayor de la ciudad. En 1634 ya se les advirtió que no construyeran más porque se diseñaría una Plaza de Armas. Las construcciones estorbaban el libre fuego de la artillería. Estas razones militares obligaron, en 1661, el traslado del colegio y de la iglesia a su definitivo emplazamiento ${ }^{46}$.

\footnotetext{
${ }^{39}$ Ibáñez Fernández/Criado Mainar, 2012: 467.

${ }^{40}$ AGNA. Sala IX, 7-1-1.Tierras de la Residencia de Montevideo... Entrega que el Padre Cosme Agullo de la residencia de San Philipe de Montevideo... otro sitio 25 por 50 hace frente a 2 calles junto al fuerte donde vive el gobernador ......otro 25 por 50 enfrente de la cuadra de la Merced....Un sitio de 50 por 50 hace frente a 2 calles y está enfrente del muelle. AGNA. Sala IX 7-1-1. Memorial de los Colegios, Hospicios y Residencias que pertenecían a los Regulares Expulsos.. En la Ciudad de Montevideo un Hospicio...

${ }^{41}$ Benedetti, 1993: 18.

${ }^{42}$ Sale, 2001: 54

${ }^{43}$ Rodríguez Gutiérrez de Ceballos, 1991: 13.

${ }^{44}$ Ibíd.: 1985: 27.

${ }^{45}$ Ibíd.: 29

${ }^{46}$ Levinton, 2012:27.
}

102 Norberto Levinton. La escena urbana como estrategia apostólica... 91 a 106. 
En cuanto a Montevideo las autoridades hicieron demoler la construcción realizada por los jesuitas, un hecho de gran contenido simbólico. En 1784 fue a Buenos Aires el Cura de la Matriz de Montevideo Presbítero Juan José Ortiz para solicitarle a Saa y Faría la confección de los planos de la nueva iglesia catedral que se quería construir. En 1790 y en conformidad con los planos de Saa y Faría se comenzó la obra. En 1794 estaba techándose. La cúpula estaba sin iniciarse, las torres carecían de sus cuerpos superiores y el frontón estaba aún por hacerse. Brambilla, ante la realidad inconclusa, trazó un dibujo de lo que iba a ser valiéndose de los planos ${ }^{47}$ (Fig. 6). Es explicativo el contraste con las edificaciones contiguas. Este integraba la expedición de Malaspina. El edificio fue finalmente rediseñado de estilo neoclásico por Tomás Toribio. Fue consagrada en 1804.

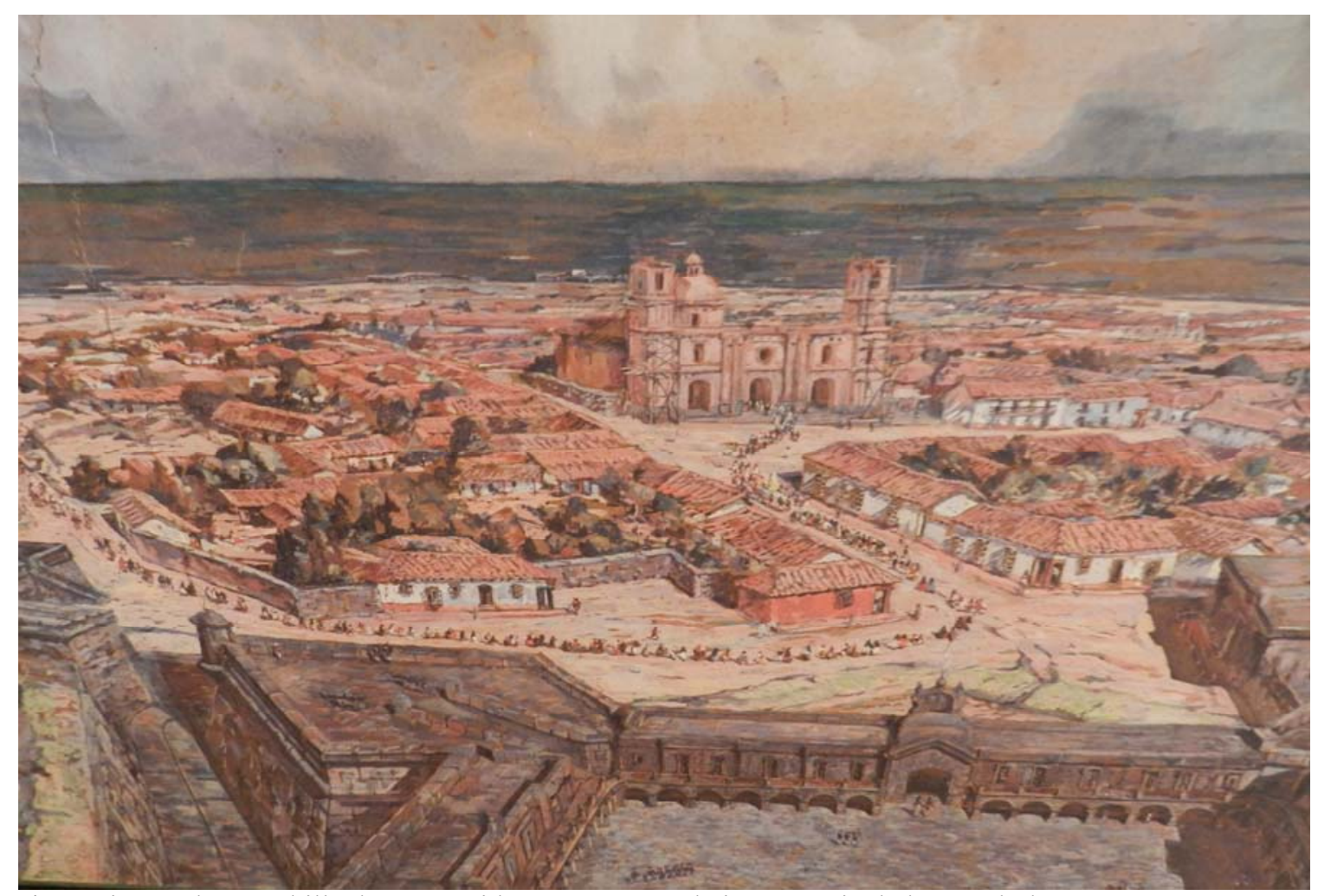

Fig. 6 Pintura de Brambilla de Montevideo que muestra la imponencia de la Catedral.

\section{Conclusiones}

Según Verdú Ruíz el historiador Chueca Goitía interpreta el uso de elementos arquitectónicos del mudéjar durante el siglo XVIII como el desarrollo de un barroco vernáculo. También designa esta estética como "barroco castizo" o "barroco mudéjar" autora interpreta que se habla del "mantenimiento de una serie de invariantes castizos, impuestos por el temperamento español, que se manifiestan en diversos períodos de la

\footnotetext{
${ }^{47}$ Fernando Brambilla 1763-1832

${ }^{48}$ Verdú Ruiz, 1998: 15, 16 y 17.
} 
historia del arte". También cita a Rodríguez Gutiérrez de Cevallos quien, según Verdú Ruíz, lo atribuye a retablistas, entalladores, escultores y ornamentadores en general que trasladaron a la arquitectura técnicas y adjetivaciones que nada tenían que ver con su esencia principal. Por eso designa como representantes a José Benito Churriguera (16651725), Teodoro Ardemans (1664-1726) y Pedro de Ribera (1681-1742).

En la obra de Antonio Forcada encontramos esta implementación del lenguaje arquitectónico regional de Aragón junto con la utilización de elementos barrocos. Esto originó cierta confusión en la historiografía de la arquitectura jesuita y misionera en relación a las atribuciones. Resultó que Kraus y Bianchi podían haber hecho bóvedas de aristas muy similares, por eso se confunde la autoría en el Colegio Máximo de Córdoba, y Bianchi con Forcada, recurrieron igualmente a las pilastras de orden toscano. Por eso es necesario apelar a los documentos que dan confiabilidad en cuanto a la presencia de cada artífice. De cualquier manera cada vez me convenzo más de la complementariedad de todos los que aportaron a estas búsquedas.

\section{Fuentes}

AGNA. Archivo General de la Nación Argentina.

\section{Bibliografía}

AECI (2004), Programa de preservación del patrimonio cultural en Iberoamérica. Proyecto Escuela Taller de Alta Gracia, Córdoba (Argentina).

Aguilar, María Dolores et. al. (1993), Mudéjar Iberoamericano. Una expresión cultural de dos mundos, Granada: Universidad de Granada.

Baracchini, Hugo y Altezor, Carlos (2010), Historia urbanística de la ciudad de Montevideo desde sus orígenes coloniales a nuestros días, Montevideo: Trilce.

Benedetti, Sandro (1993), Fuori dal clasicismo, Roma: Bonsignori Editore.

Buschiazzo, Mario J. (1940), La Estancia Jesuítica de Santa Catalina, Buenos Aires: Academia Nacional de Bellas Artes.

(1969), Estancias Jesuíticas de Córdoba, Buenos Aires: Filmediciones Valero.

Carmona, Liliana y Gómez, María Julia (1999), Montevideo: proceso planificador y crecimientos, Montevideo: Instituto de Historia de la Arquitectura de la Facultad de Arquitectura de la Universidad de la República. http://es.scribd.com/doc/124926647/Montevideo-Proceso-Planificador-yCrecimientos-Carmona-Nacional\#scribd

Carretero Calvo, Rebeca (2012), Arte y arquitectura conventual en Tarazona en los siglos XVII y XVIII, Tarazona: Centro de Estudios Turiasonenses.

Chueca Goitía, Fernando (1985), Historia de la arquitectura occidental. Barroco en España, Madrid: Dossat. 
Ferres, Carlos (1975), Época Colonial. La Compañía de Jesús en Montevideo, Montevideo: Ministerio de Educación y Cultura.

Fraga González, María del Carmen (1977), La arquitectura Mudéjar en Canarias, Santa Cruz de Tenerife: Aula de Cultura de Tenerife.

Furlong SJ, Guillermo y Buschiazzo, Mario J. (1943), “Arquitectura Religiosa Colonial”. Archivum, Revista de la Junta de Historia Eclesiástica Argentina, Tomo I-Cuaderno 2. Buenos Aires, pp. 450 a 471.

Furlong SJ, Guillermo (1946), Arquitectos argentinos durante la dominación hispánica, Buenos Aires: Huarpes S.A.

(1959), "Algunos planos de Iglesias y Colegios de la Compañía de Jesús en España”, Archivum Historicum Societatis Iesu, XXVIII, 55, Roma, IHSI, pp. 205208 y ss. sin numerar.

Gallardo, Rodolfo (1978), La Arquitectura religiosa en Córdoba en el período hispánico, Córdoba: Gobierno de la Provincia de Córdoba.

Golvin, Lucien (1970), Essai sur l'architecture religieuse Musulmane, Paris: Klincksieck.

Gómez-Ferrer, Mercedes (2012), "La arquitectura jesuítica en Valencia. Estado de la cuestión", Actas del Simposio Internacional la Arquitectura Jesuítica. Coord. por María Isabel Alvaro Zamora, Javier Ibáñez Fernández y Jesús Criado Mainar. Zaragoza (España), Universidad de Zaragoza, pp. 355 a 392. Ifc.dpz.es/recursos/publicaciones/31/96/12gomezferrer.pdf/

Grenón SJ, Pedro (1963), Un plano histórico de la Universidad, Córdoba: Imprenta de la Universidad Nacional de Córdoba.

Guidi, Freddy F., Sassi, María Teresa y Naselli, Cesar (1987), "La estancia de Jesús María”, en: Arquitectura Colonial Argentina, Buenos Aires: Ediciones Summa.

Ibáñez Fernández, Javier y Criado Mainar, Jesús (2012), "La arquitectura jesuítica en Aragón. Estado de la cuestión". Actas del Simposio Internacional la Arquitectura Jesuítica. Coord. por María Isabel Alvaro Zamora, Javier Ibáñez Fernández y Jesús Criado Mainar. Zaragoza (España), Universidad de Zaragoza, pp. 393 a 472.

Lambert, Elie (1933), “L'art Mudéjar”. Gazette des Beaux Arts, IX, pp. 17-33.

Lascano González, Antonio (1941), Monumentos religiosos de Córdoba Colonial. Buenos Aires: Amorrortu.

Levinton, Norberto (1999), "El aporte Mudéjar del Hermano Forcada a la arquitectura de Córdoba", en Congreso Internacional Jesuitas 400 años en Córdoba, Tomo 2, Córdoba; Universidad Nacional de Córdoba y Junta Provincial de Historia de Córdoba, pp. 187 a 200.

(2012), Un espacio privilegiado para la Compañía de Jesús, Arquitectura de la Compañía de Jesús en Buenos Aires, Buenos Aires: Contratiempo Ediciones.

Lugones, Leopoldo (1973), El Imperio Jesuítico, Buenos Aires: EUDEBA. 
Mörner, Magnus (1971), "Un procurador jesuita del Paraguay ante la corte de Felipe V". Separata de Historiografía y bibliografía americanistas. Vol. XV, No3. Sevilla, diciembre, pp. 367 a 443.

Navarro Catalán, David Miguel (2012). "La construcción de la parroquial de San Carlos de Ontinyent", Boletín de la Sociedad Castellonense de Cultura, Tomo LXXXVIII. Enero-Diciembre, pp. 371 a 382.

Nicolini, Alberto y Paterlini de Koch, Olga (1974), "Nuestra Señora de Alta Gracia. Los Jesuitas en Córdoba", DANA, $\mathrm{N}^{\circ} 2$. Resistencia.

Page, Carlos (1999), La Manzana Jesuítica de la Ciudad de Córdoba, Córdoba: Eudecor. (2000), La Estancia Jesuítica de Alta Gracia, Córdoba: Universidad Católica de Córdoba y Universidad Nacional de Córdoba.

Rodríguez Gutiérrez de Ceballos, Alfonso SJ. (1985), Estudios del barroco salmantino. El Colegio Real de la Compañía de Jesús (1617-1779), Salamanca: Centro de Estudios Salmantinos.

(1991), La Plaza Mayor de Salamanca, Salamanca: Centro de Estudios Salmantinos.

Sale, Giovanni (2001), Pauperismo architettonico e architettura gesuitica, Milano: Editoriale Jaca Book.

Sobrón SI, Dalmacio H. (1997), Giovanni Andrea Bianchi, Buenos Aires: Ediciones Corregidor.

Storni SI, Hugo (1980), Catálogo de los jesuitas de la Provincia del Paraguay (Cuenca del Plata) 1585-1768, Roma: IHSI.

Vázquez Barrado, Ana (1994), De arquitectura jesuítica, Roma: Accademia Spagnola di Storia, Archeología e Belle Arti.

Verdú Ruíz, Matilde (1998), El arquitecto Pedro de Ribera (1681-1742). Madrid, Instituto de Estudios Madrileños.

Watt, W. Montgomery (1981), Historia de la España islámica, Madrid: Alianza Editorial. 